\title{
Belphégor
}

\section{Compositeurs mis en page : Vedettes et génies dans Musica et Le Mercure musical}

\section{Marie-Pier Leduc}

\section{(2) OpenEdition}

\section{Journals}

Édition électronique

URL : https://journals.openedition.org/belphegor/4157

DOI : 10.4000/belphegor.4157

ISSN : 1499-7185

Éditeur

LPCM

\section{Référence électronique}

Marie-Pier Leduc, «Compositeurs mis en page : Vedettes et génies dans Musica et Le Mercure musical », Belphégor [En ligne], 19-2 | 2021, mis en ligne le 04 janvier 2022, consulté le 20 septembre 2022. URL : http://journals.openedition.org/belphegor/4157 ; DOI : https://doi.org/10.4000/belphegor. 4157

Ce document a été généré automatiquement le 20 septembre 2022.

Creative Commons - Attribution - Pas d'Utilisation Commerciale - Pas de Modification 4.0 International - CC BY-NC-ND 4.0

https://creativecommons.org/licenses/by-nc-nd/4.0/ 


\title{
Compositeurs mis en page : Vedettes et génies dans Musica et Le Mercure musical
}

\author{
Marie-Pier Leduc
}

1 Le lecteur ou la lectrice résidant à Paris au début du XXe siècle qui s'intéressait à la musique avait l'embarras du choix pour s'informer. Un éventail de publications spécialisées était offert, en plus des rubriques musicales paraissant dans les journaux quotidiens ou dans les diverses revues culturelles, théâtrales, littéraires ou généralistes. Les éléments trouvés dans ces différents supports sont parfois radicalement différents, du choix des sujets qui seront ou non traités à la présentation visuelle, en passant par le ton employé. Si ces variations sont en partie imputables aux musicographes - critiques, essayistes, historiens et vulgarisateurs - qui ont chacun leurs propres champs de compétences, préférences esthétiques et styles, elles sont aussi le fruit de choix éditoriaux faits par les organes de presse, ne serait-ce que dans la sélection des collaborateurs - artisans et auteurs - qui «font », collectivement, les périodiques.

2 Musica, mensuel fondé en 1902 par l'éditeur Pierre Lafitte et l'impresario Gabriel Astruc, se distingue substantiellement des autres publications de l'époque. Il innove en délaissant la formule éprouvée de la «revue » au profit d'une formule «magazine », inédite jusque-là en France dans le domaine musical. Le créneau du magazine illustré avait été développé par Lafitte dès 1898 suivant le modèle anglo-saxon, d'abord dans le domaine sportif, avec le mensuel (puis hebdomadaire) La Vie au Grand Air ${ }^{1}$ où le discours est formé par l'association d'illustrations et de textes (titres, légendes, chapeaux et le texte lui-même) dans une maquette complexe ${ }^{2}$; Lafitte applique une formule similaire dans des sphères aussi diversifiées que la presse féminine avec Femina, le «country life français " avec Fermes \& Châteaux et la vulgarisation des savoirs avec le magazine familial Je sais tout. La mise en page de Musica, tout comme celle de La Vie au Grand Air, invite à une consommation par le «feuilletage » transformant le lecteur ou la lectrice de l'époque en spectateur ou spectatrice qui reçoit un message tout en étant diverti ${ }^{3}$. 
Rien n'empêche cependant une lecture attentive de Musica qui propose une variété de genres journalistico-musicographiques (portraits, entretiens, enquêtes, textes de vulgarisation ou pédagogiques, critiques, etc.) sur une diversité de sujets; certains textes sont en phase avec l'actualité de la vie musicale (opéras représentés, concerts, décès d'un musicien, etc.), tandis que d'autres s'en détachent complètement. L'examen des légendes et des chapeaux introductifs ajoutés à plusieurs textes et qui se distinguent visuellement par l'usage de l'italique permet de cerner les éléments que la rédaction souhaite faire ressortir pour le lecteur ou la lectrice qui ne choisirait que de feuilleter le magazine. Il apparaît évident que Musica mise sur le vedettariat et présente principalement des artistes jouissant déjà d'une certaine célébrité. Le vedettariat en musique est bien antérieur au $\mathrm{XX}^{\mathrm{e}}$ siècle, tel qu'en témoigne l'engouement pour les virtuoses et plus particulièrement pour les castrats dès le début $\mathrm{du}$ XVIII ${ }^{\mathrm{e}}$ siècle ainsi que pour les chanteurs d'opéra en général ${ }^{4}$. L'innovation du magazine de Lafitte et Astruc réside dans l'application systématique du concept de vedette à l'ensemble des acteurs de la vie musicale, y compris les compositeurs dont le statut avait jusque-là principalement été régi par l'opposition artisan/génie issue de la fin du XVIII ${ }^{\mathrm{e}}$ siècle et du XIX ${ }^{e}$ siècle.

3 Les transformations du statut de l'artiste ont été étudiées par Nathalie Heinich qui propose une analyse de l'évolution du statut des élites culturelles s'échelonnant du début du XIX ${ }^{e}$ siècle jusqu'à nos jours ${ }^{5}$. L'émergence au XIX siècle d'une nouvelle élite " en marge"- les artistes romantiques (géniaux, bohèmes, maudits, etc.) - est intimement liée à l'assimilation, après la Révolution française, de l'excellence comme découlant du talent, formé à la fois du don inné et du travail acharné. Conformément au nouvel idéal d'égalité du "régime démocratique », la grandeur est compensée voire payée - par la marginalité. Un changement de valeurs s'opère au $\mathrm{XX}^{\mathrm{e}}$ siècle avec la transformation du "régime médiatique " déjà initié au XVIII ${ }^{e}$ siècle ${ }^{6}$ mais qui se renouvelle et monte en puissance avec la duplication de plus en plus massive de l'image. La grandeur devient alors liée à la possession d'un « capital de visibilité $»^{7}$ tout en se nourrissant de récits, de discours et de textes ${ }^{8}$. Musica illustre parfaitement ce changement de paradigme au début $\mathrm{du} \mathrm{XX}^{\mathrm{e}}$ siècle dans la sphère musicale, et $\mathrm{y}$ participe activement avec sa formule novatrice.

Les compositeurs dans Musica ont récemment fait l'objet d'un article de Michel Duchesneau' qui a démontré comment plusieurs d'entre eux se sont prêtés au jeu médiatique du magazine en se laissant photographier, en accordant des entretiens ou y en prenant la plume. Dans cet article, nous nous intéressons plus particulièrement à la présentation des compositeurs vivants comme de véritables vedettes dans Musica, en lien avec la formule «magazine » adoptée par la publication. Nous proposons aussi de comparer le cas de Musica au Mercure musical, une "petite revue " proche de l'avantgarde fondée en 1905 par Louis Laloy et Jean Marnold qui met principalement en avant des artistes émergents, souvent en marge ou en conflit avec les institutions officielles, en utilisant une rhétorique avant-courrière qui annonce les génies qui seront un jour reconnus. Cette comparaison permet d'illustrer comment le statut accordé aux compositeurs peut varier dans deux périodiques qui sont contemporains mais avec des formats, des réseaux de collaborateurs et des lectorats cibles radicalement différents. Ce sont ces particularités qu'il convient de présenter dans un premier temps. 


\section{Derrière et devant le périodique : le réseau de collaborateurs et le lectorat de Musica et du Mercure musical}

\section{Musica, à l'intersection des méthodes de Pierre Lafitte et du réseau de Gabriel Astruc}

5 Musica et Le Mercure musical proposent tous les deux des articles développés et une mise en page soignée, mais leurs choix éditoriaux ne pourraient être plus opposés. Les forces derrière la création des deux périodiques contrastent à bien des égards. Musica porte la signature visuelle des publications de la maison Lafitte, dont il est le troisième titre après La Vie au Grand Air (1898-1914 ; 1916-1922) et Femina (1901-1939). Lafitte lance ensuite coup sur coup plusieurs autres publications illustrées, toutes sur papier couché: le magazine encyclopédique illustré Je sais tout (1905-1939) - dans lequel paraissent notamment en feuilletons les aventures d'Arsène Lupin de Maurice Leblanc -, Fermes \& Châteaux (1905-1914), Jeunesse qui sera renommé Le Petit Magazine de la jeunesse (1905-1907) et l'hebdomadaire d'information J'ai vu (1914-1920) ${ }^{10}$. On lui doit aussi le quotidien Excelsior (1910-1940) qui révolutionne la presse française par l'adaptation du modèle de construction de la page développé dans ses magazines au journal $^{11}$, ainsi que plusieurs collections de livres. En raison de difficultés financières aggravées par la guerre, Lafitte vend ses titres en 1916.

6 L'initiateur de Musica n'est pas Lafitte mais son cofondateur Gabriel Astruc, comme ce dernier le rapporte dans ses mémoires : «J'eus l'idée de lancer sous l'égide de Femina un autre magazine, musical, celui-là. L'idée plut à Lafitte et à ses commanditaires [...]. J'établis la maquette des six premiers numéros du futur journal. Lafitte approuva mon plan $»^{12}$. Avant de fonder Musica, Astruc avait cumulé diverses fonctions journalistiques et éditoriales lui faisant côtoyer le Tout-Paris. Il avait été actif comme critique ou chroniqueur pour Le Succès, L'Événement, Le Figaro et Le Gaulois, généralement sous le pseudonyme anagrammatique «Surtac $»^{13}$. Du côté de son expérience éditoriale, il avait d'abord fait un bref séjour à la maison d'édition de partitions de son cousin et futur beau-père Wilhelm Enoch, Enoch frères \& Costallat, avant d'entrer chez l'éditeur littéraire Paul Ollendorff en 1882. Astruc retourne à l'édition musicale en 1897 lorsque Wilhelm Enoch, dont la fille Marguerite était alors sa fiancée, lui propose de prendre la place de son ancien associé14. Lorsqu'il contacte Lafitte pour fonder Musica, les responsabilités d'Astruc chez Enoch consistent «à éditer de la musique, à "auditionner" des Prix de Rome, à faire des maquettes de titres américains, avec l'ambition d'élargir, au théâtre, au concert, dans la presse, le champ d'action de la Maison ${ }^{15}$. Astruc quitte ensuite Enoch pour fonder la Société musicale G. Astruc, agence artistique et maison d'édition de partitions, qu'il établit au Pavillon de Hanovre, boulevard des Italiens, en avril 1904. La Société musicale organise plusieurs événements privés - fêtes et soirées qui agissent comme des laboratoires culturels pour la haute société parisienne (dont plusieurs membres contribuent financièrement à ses projets) ainsi que des galas et levées de fonds pour le compte d'une tierce personne. Le cœur des activités de la Société musicale d'Astruc est cependant l'organisation d'événements publics. On lui doit la Grande Saison de Paris qui a accueilli de 1905 à 1913 les artistes parmi les plus recherchés du moment, ainsi que le Théâtre des ChampsÉlysées ${ }^{16}$. Les différents projets d'Astruc ont régulièrement dépendu du soutien 
financier de mécènes, dont certains, comme le comte Isaac de Camondo et Henry Deutsch de la Meurthe ${ }^{17}$, s'adonnaient à la composition, et leurs œuvres étaient en retour éditées par la Société musicale et jouissaient d'une certaine visibilité dans Musica et son supplément musical. Le réseau d'artistes professionnels représentés par Astruc était varié : chanteurs et chanteuses (Marguerite Carré, Lucienne Bréval et Ernest Van Dyck), instrumentistes (Jacques Thibaud, Eugène Ysaÿe, Pablo Casals, Arthur Rubinstein, Alfred Cortot, Wanda Landowska), chefs d'orchestre (Édouard Colonne et Félix Weingartner) et compositeurs (Gabriel Fauré, Camille Saint-Saëns et Richard Strauss) ${ }^{18}$. Les pages de Musica s'alimentent en bonne partie au réseau professionnel d'Astruc; ses intérêts sont doublement servis puisque la revue offre une tribune de choix à plusieurs de ses artistes. Dans ses mémoires, Astruc qualifie d'ailleurs Musica de « magazine musical richement subventionné par les artistes et les impresarii $»^{19}$.

7 Fort de son réseau dans le domaine musical déjà bien construit en 1902 et qui ne cessera de croître, c'est Astruc qui se charge de la « direction artistique » de Musica en collaboration avec Charles Joly (rédacteur en chef), Pierre Lalo, Henri de Curzon, Ernest Van Dyck ainsi que Robert Brussels et Georges Pioch qui agissaient tous les deux comme secrétaires de rédaction. Après le décès prématuré de Joly en 1905, Pioch prend en charge le poste de rédacteur en chef jusqu'en 1910. Lui succède ensuite le compositeur Xavier Leroux de novembre 1910 jusqu'à la fin de la publication en août $1914^{20}$. Avec son équipe, Astruc supervise à la fois le contenu et « le puzzle de la typo et des clichés [qui] est un jeu difficile", car "chaque page possède l'ordonnance architecturale et le style symétrique d'un monument bien équilibré $»^{21}$.

8 Lafitte semble s'être davantage occupé de la mise en marché et de la publicité, abondante dans les pages de Musica, comme dans tous les autres titres de la maison, variant de quatre à parfois plus de dix pages par numéro sur seize à vingt pages de contenu. Le prix de l'abonnement annuel (12 francs) et du numéro individuel (1 franc ou 2 francs pour les numéros spéciaux dont le supplément musical est exceptionnellement volumineux) est particulièrement abordable pour une publication aussi richement illustrée, surtout si l'on ajoute les primes à la souscription à l'effigie du magazine ${ }^{22}$ qui valent souvent plus que l'abonnement lui-même. La tactique est simple : plus la revue se vend, plus Lafitte peut compter sur des revenus publicitaires. Les autres publications de Lafitte sont évidemment bien annoncées. Pour faire connaître son nouveau-né musical, Lafitte le distribue d'abord gratuitement au lectorat de Femina en l'encartant dans son périodique féminin ${ }^{23}$. L'homme d'affaires offre aussi des réductions de tarifs pour l'abonnement à plusieurs de ses périodiques et négocie pour ses abonnés des arrangements avec divers commerces ${ }^{24}$. Lafitte va plus loin dans la convergence de ses projets. Il ouvre dès le mois de décembre 1902 une "salle d'exposition" au 9, avenue de l'Opéra - dans laquelle "sont concentrés tous les services de Musica, de Femina et de La Vie au Grand Air » avec une "entrée libre pour [les] lectrices et abonnées $»^{25}$-, avant de déménager en 1907 au 90, avenue des ChampsÉlysées. En plus des services d'administration et de rédaction, il y fait construire une salle de théâtre de près de 600 places convertible en salle de bal (qui est peu après inaugurée sous le nom de Théâtre Femina ${ }^{26}$ ) et une galerie des fêtes - toutes deux pouvant être louées par des particuliers - ainsi qu'un atelier et un salon de "photographie d'art $»^{27}$. Y sont organisés, sous la direction d'Astruc, les concerts Femina-Musica qui étaient auparavant tenus à l'Automobile-Club de France ${ }^{28}$ et autres matinées musicales qui réunissent "les noms des grands artistes [...] et de nos compositeurs les plus réputés $»^{29}$ ainsi que de nombreux concours organisés par le 
magazine. L'édifice abrite aussi, à partir d'octobre 1907, le Conservatoire Femina-Musica dirigé par Xavier Leroux ${ }^{30}$. Moderne, la société de Lafitte multiplie ses activités et ses produits et augmente par le fait même sa visibilité.

\section{Petite bourgeoisie, femmes et amateurs : le contenu fait sur mesure pour le lectorat cible de Musica}

Bien que ce soient principalement Astruc et Joly qui supervisent le contenu et le processus éditorial de Musica, Lafitte avait bien entendu son mot à dire. Chaque numéro de Musica est accompagné d'un supplément musical d'environ 24 pages, l'Album-Musica, constitué principalement de pièces pour piano ou voix et piano d'un niveau de difficulté peu élevé. Les morceaux sont sélectionnés par un «jury » formé par l'équipe d'Astruc, dont les goûts semblent avoir été trop élitistes aux yeux de Lafitte :

Messieurs, disait-il, je regrette de constater que vous avez une tendance déplorable, dans vos sélections, à oublier Celle qui devrait être toujours présente à vos pensées. J'ai nommé l'Épicière de Carpentras. Partez de ce principe, que le caviar et les ortolans sont faits pour l'élite, c'est-à-dire pour 1500 personnes. Si nous voulons atteindre les gros tirages, travaillons pour la Mercière de Draguignan ou sa cousine la Receveuse des Postes de Pont-à-Mousson. Il faut que dans chaque ville de province, quand une jeune fille se met au piano, toute la famille s'écrie en chœur : "Comme c'est joli! " et que la Maman, interrogée sur l'origine dudit morceau, réponde invariablement : «Elle a trouvé ça dans Musica. ${ }^{31}$

Ce commentaire condense l'image du lectorat auquel Lafitte destine prioritairement son magazine, soit un lectorat issu de la petite bourgeoisie ${ }^{32}$, essentiellement féminin, pratiquant la musique en amateur. C'est ce vaste bassin de lecteurs et lectrices, plutôt que l'« élite [de] 1500 personnes ", qui permet à Musica d'atteindre un tirage important qui se situe entre 45000 et 50000 exemplaires dès novembre $1903^{33}$.

11 Le contenu de Musica semble le résultat d'une constante négociation entre les intérêts et les goûts de Lafitte et d'Astruc. Le magazine présente des entretiens, des portraits biographiques ainsi que des articles de vulgarisation sur divers sujets. Qu'il soit question d'un artiste, d'une mise en scène d'opéra, des concours de fin d'année du Conservatoire, d'une institution d'enseignement, de la facture instrumentale ou d'une période de l'histoire de la musique, chaque texte est richement illustré, le plus souvent par des montages photographiques élaborés. En faisant intervenir massivement la photographie, Musica investit le marché de la petite bourgeoisie et propose d'instruire et de divertir le lectorat qui en est issu.

L'illustration n'est pas la seule tactique utilisée pour divertir le lectorat, l'« enfantpublic » que les "beaux livres d'images" comme Musica sont "faits pour amuser", selon les mots d'Astruc ${ }^{34}$. Les potins et faits divers - qui sont «les plus purs représentants de la culture de masse $»^{35}$ - ponctuent à quelques reprises les pages $d u$ magazine. C'est notamment le cas avec la publication en 1903 de la pièce La Fuite de la princesse Louise de Saxe, dont la vie privée faisait alors les manchettes en raison d'un scandale d'adultère. La Rédaction précise qu'elle a «cru qu'il était intéressant de faire connaître [aux] lecteurs ce curieux morceau qui est d'une musicalité très distinguée ${ }^{36}$. Un esprit similaire se retrouve dans la présentation d'une reproduction autographe d'un exercice musical de Maria Daurignac dont l'importante famille était alors sur la sellette en raison d'un scandale financier impliquant de lourdes dettes: "Cette découverte est trop amusante pour que nous en privions nos lecteurs, à qui nous 
offrons la primeur inédite d'un manuscrit choisi par nous dans les "cahiers de notes" de Maria Daurignac $~^{37}$. L'inventivité de Musica va encore plus loin avec les jeux-concours dans l'esprit des mots croisés et jeux des différences de nos journaux actuels - tels que le «carré magique musical », le " compositeur coupé en 40 morceaux » à reconstituer et les devinettes du type «Quel est cet air? Quel est son auteur?", avec des prix à la clef $^{38}$.

13 Musica se démarque aussi de reste de la presse musicale de l'époque par ses nombreux articles à vocation pédagogique destinés à accompagner les apprentis musiciens. Certains de ceux-ci recourent à l'illustration pour rendre plus concrètes les explications.

III. 1. « Les positions du pianiste » (Musica, octobre 1902, pp. 13-14)
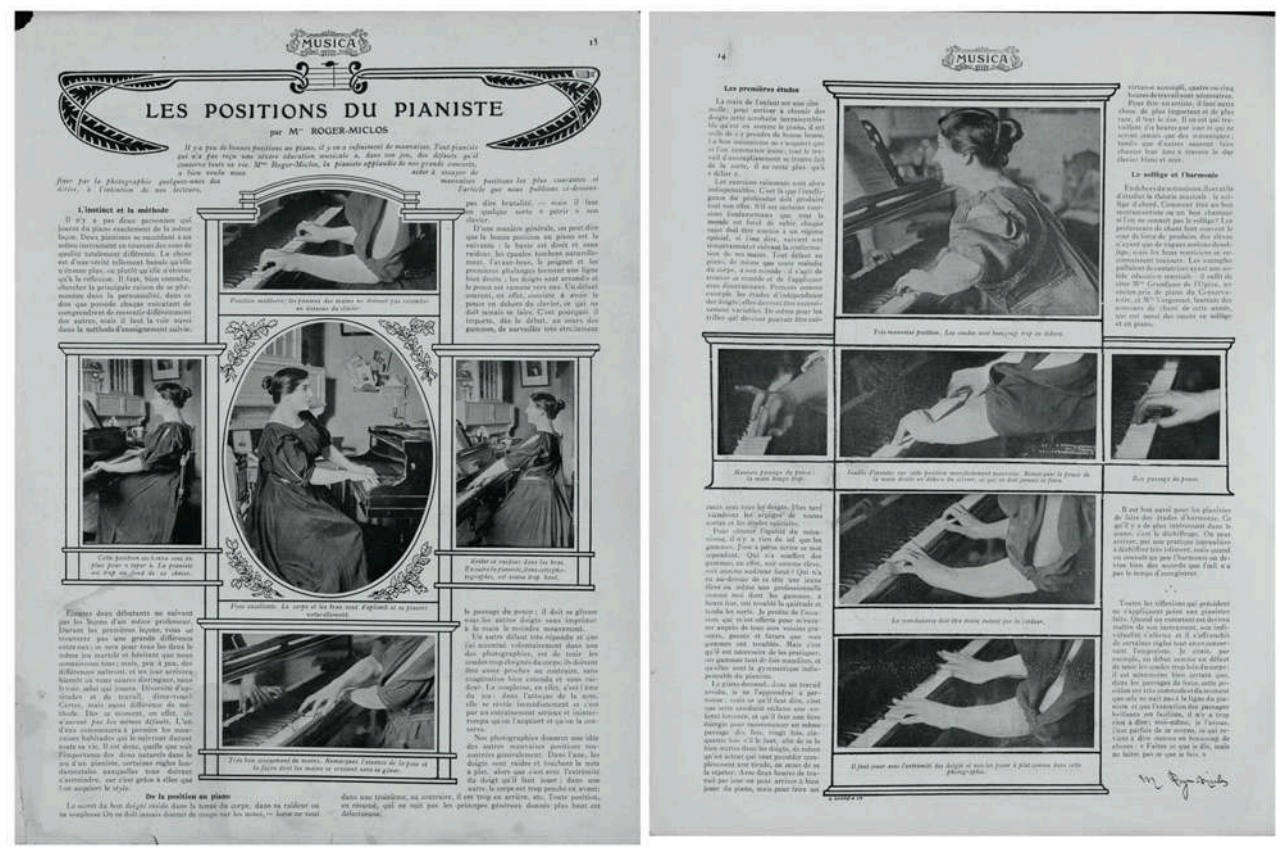

L'Album-Musica est systématiquement accompagné d'une rubrique "Conseils pour l'exécution de nos morceaux » jusqu'au numéro de novembre 1910. Musica expérimente aussi dans ses quatre premiers numéros une rubrique "Cours d'enseignement musical » afin de «mettre ces questions d'enseignement à la portée de tous, à la portée surtout des mères de famille qui pourront ainsi surveiller l'éducation de leurs enfants ", la musique étant « devenue en quelque sorte l'ornement de la famille " ${ }^{39}$.

Le magazine présente ainsi un côté familial et pratique et agit, à l'instar de la presse féminine en général, comme un "puissant instrument de maintien du conformisme, de la division du travail et des rôles entre les sexes $»^{40}$.

\section{Le Mercure musical, une revue par et pour l'avant-garde}

Le Mercure musical se situe à l'opposé du modèle d'affaires de Musica. Le nouveau bimensuel musical (qui deviendra mensuel) a vu le jour sous l'« illusoire patronage $»^{41}$ du Mercure de France, la plus grande des "petites revues" littéraires de l'époque. Le Mercure musical emprunte plusieurs éléments de la maquette et la structure bipartite de son aînée littéraire, mais adopte un ton bien plus combatif mis au service de l'avant- 
garde musicale incarnée par Claude Debussy et Maurice Ravel ${ }^{42}$. L'un des cofondateurs du Mercure musical, Jean Marnold, était le critique musical du Mercure de France. L'autre cofondateur, le critique et érudit Louis Laloy, avait pour sa part claqué la porte de la Revue d'histoire et de critique musicales car il la trouvait «trop modérée $»^{43}$.

«Revue d'esthètes", Le Mercure musical propose des textes musicologiques (histoire, ethnomusicologie ${ }^{44}$, théorie), des contributions littéraires ainsi que des analyses ou critiques arrimées à l'actualité musicale ${ }^{45}$. La frontière entre les différents genres est poreuse, et une critique peut par exemple prendre la forme d'une satire dialoguée. Plutôt que d'exploiter la photographie comme Musica, Le Mercure mise sur le dessin et la gravure, options moins coûteuses, en publiant à l'occasion des croquis de Charles Léandre et des "compositions inédites pour Simone» de Georges d'Espagnat qui accompagnent des poèmes de Rémy de Gourmont. Leur caractère esquissé offre un pendant visuel aux aphorismes et autres « pensées » qui ponctuent le périodique.

III. 2. Charles Léandre, «Croquis d'album : Ricardo Viñes » et «Croquis d'album : Déodat de Séverac » (Le Mercure musical, 15 juillet 1905 et $1^{\text {er }}$ février 1906).


Une part importante des textes du Mercure musical sont de la plume de Laloy et Marnold ${ }^{46}$. Ils s'entourent aussi d'auteurs aux horizons variés : des littéraires comme Rémy de Gourmont (qui publie même une mélodie) et Colette, des musicologues tels que Pierre Aubry et Lionel de La Laurencie et des critiques professionnels. Certains portent plusieurs chapeaux, comme Romain Rolland : ce dernier publie à la fois des études musicologiques, des critiques et des extraits de La Révolte, quatrième volume de son roman Jean-Christophe. Le Mercure est la principale chambre d'écho des idées des Apaches, un groupe de jeunes musiciens et artistes se réunissant de façon informelle qui comprend alors Maurice Ravel, Déodat de Séverac, Ricardo Viñes, Désiré-Émile Inghelbrecht, Paul Ladmirault, Léon-Paul Fargue, Paul Sordes, Maurice Delage, Florent Schmitt, Tristan Klingsor, Émile Vuillermoz, entre autres. Environ du même âge, plusieurs se connaissaient du Conservatoire de Paris et plus spécifiquement de la classe 
de composition de Gabriel Fauré. Liés par l'amitié et des idées esthétiques communes, ils s'intéressent à l'art non occidental (et plus particulièrement l'art russe et asiatique) et au symbolisme. Le groupe considère Debussy (qui a toujours gardé ses distances avec les Apaches) comme un "prophète " et forme "une garde d'honneur» autour de Ravel ${ }^{47}$.

Bien qu'ils ne fassent pas partie des Apaches, Laloy et Marnold sont animés par le même idéal esthétique ${ }^{48}$. Une amitié étroite lie d'ailleurs Laloy à Debussy depuis 1902, et Marnold, proche de Ravel, est alors l'hôte de plusieurs des réunions des Apaches. Les auteurs du Mercure musical ne parlent certes pas d'une seule et même voix, mais une part importante des collaborateurs de la revue est à l'évidence issue du cercle élargi des Apaches ou est sympathique à leurs orientations. La solidarité envers Ravel se manifeste dès le premier numéro du Mercure, avec la publication dans la section «Échos» d'un message d'encouragement signé «Pan» (probablement Vuillermoz) pour la cinquième tentative de Ravel au prix de Rome : « CONCOURS DE ROME. - M. Maurice Ravel a l'intention de se présenter cette année à ce concours. Tous nos vœux l'accompagnent ! $»^{49}$. En novembre de la même année, les « Échos » annoncent les cours de composition de Ravel en termes élogieux : «M. Maurice Ravel reprend ses cours et leçons de composition, chez $\mathrm{M}^{\text {lles }}$ Chaigneau, 162, avenue Victor-Hugo. On sait trop ce que nous pensons de lui pour que nous ajoutions à son nom, déjà illustre, un seul mot de recommandation $»^{50}$. Vuillermoz - critique à la subversive ironie, «musicien de combat » des Apaches selon Delage ${ }^{51}$ et que Ravel, dans un esprit humoristique, qualifie $d^{\prime}$ ' archange protecteur de notre petite chapelle $»^{52}$ - aurait été la cheville ouvrière du Mercur $^{53}$. Il a probablement orchestré la publication du poème de Ravel pour sa mélodie Noël des jouets peu avant la première de l'œuvre en mars $1906^{54}$, et il y publie certains de ses textes les plus corrosifs, signés tantôt Willy ou Henry Gauthier-Villars, dont il était l'un des principaux ghostwriters, tantôt de son propre nom. Vuillermoz est l'auteur du dialogue fictif entre un vieillard et un jeune homme à propos de la modernité (signé Willy), « déclaration d'une guerre musicale ${ }^{55}$ contre Vincent d'Indy et la Schola cantorum qui ouvre le premier numéro du Mercure musical et qui donne le ton à la revue. Plusieurs Apaches (Vuillermoz, Ravel et Schmitt) font d'ailleurs partie des membres fondateurs de la Société musicale indépendante lancée en 1910 pour protester contre la Société nationale de musique alors dirigée par d'Indy et jugée trop conservatrice et nationaliste dans le choix d'œuvres jouées au concert. Le refus de partitions de Ravel et Delage provoqua le schisme ${ }^{56}$.

L'éditeur du Mercure, Eugène Demets, «qui aimait trop la musique nouvelle pour faire fortune $»^{57}$, était un éditeur de partitions et organisateur de concerts tout comme Astruc. Demets publie plusieurs des œuvres de Ravel jusqu'à Miroirs (1906) ${ }^{58}$. Trois œuvres de Séverac et trois de Ravel sont d'ailleurs les seules à occuper l'espace publicitaire de Demets sur la $2^{\text {e }}$ de couverture pour les numéros 3 à 10 , ce qui témoigne bien de la visibilité que l'on souhaite donner aux œuvres des jeunes compositeurs des Apaches.

Les auteurs du Mercure musical semblent s'adresser avant tout à leurs pairs, soit un lectorat issu de l'élite artistique, littéraire et intellectuelle. Les quelques publicités que comprennent les numéros révèlent que la revue est diffusée à la fois dans les cercles de l'avant-garde musicale, de la musicologie et de l'ethnomusicologie ainsi que dans les cercles littéraires et plus particulièrement symbolistes. On retrouve annoncés en $4^{\mathrm{e}}$ de couverture le Mercure de France, L'Ermitage et L'Euvre internationale et plus tard la Revue 
générale de bibliographie française. D'autres pages publicisent La Rénovation esthétique, Le Monde artiste, La Revue du mois, Antée (Bruxelles), L'Art moderne (Bruxelles) et The Musiclovers Calendar (Boston) dont Laloy est le correspondant parisien. On retrouve aussi quelques publicités pour des concerts de musique moderne, des ouvrages savants en vente au bureau du Mercure et des éditions de partitions nichées comme les «Extraits des Maîtres musiciens de la Renaissance française " publiés par Henry Expert chez Leduc ${ }^{59}$ et " Musique arabe \& maure » publiée chez Edmond Yafil à Alger.

Le Mercure musical, qui jouit d'une forte légitimité culturelle et dont « une élite seule pouvait goûter la généreuse ardeur combative et la rectitude de jugement $»^{60}-$, n'est cependant pas rentable. Laloy éponge lui-même le déficit jusqu'à ce que l'occasion se présente de transformer la revue en organe de la Section française de la Société internationale de musique (SIM), que Jules Écorcheville cherchait justement à doter d'une publication ${ }^{61}$. À partir de 1907, Écorcheville remplace Marnold comme codirecteur de la nouvelle mouture du Mercure qui prendra le nom de Revue musicale SIM. Le périodique continue à publier un mélange d'études musicologiques et de critiques, mais la part littéraire et le ton combatif sont laissés de côté. Vuillermoz, qui s'est brouillé avec Laloy sur la question du « debussysme » en $1907^{62}$, est totalement absent du nouveau périodique jusqu'en 1910 (Laloy en avait quitté la codirection en 1909); il effectue un retour comme collaborateur régulier en 1911 et devient son rédacteur en chef en 1912. Vuillermoz se retient cependant de tout coup d'éclat, respectant la volonté d'Écorcheville de "maintenir la concorde et l'harmonie », pour reprendre les mots que le directeur adresse à son rédacteur en chef au moment de partir pour la guerre ${ }^{63}$. Ainsi, malgré le retour de Vuillermoz, la Revue musicale SIM n'a plus beaucoup en commun avec Le Mercure musical initial où le choc des idées était recherché, comme annoncé par Laloy dans son programme inaugural en mai 1905 : « Il y aura des dissonances dans notre concert. Loin de les éviter, nous les chercherions plutôt, sachant que du choc des opinions contraires se dégage ce qui est, pour chacun de nous, la vérité. Seule une sincérité égale nous unit et fera, je l'espère, notre force ${ }^{64}$. Cet esprit combatif a fait la singularité du Mercure musical des années 1905-1906, qui suit le modèle des nombreuses «petites revues» de combat qui sont nées à la fin du $\mathrm{XIX}^{\mathrm{e}}$ et au début du XX' $\mathrm{Xiècle}^{65}$.

\section{Vedettes et génies, quel statut pour la légitimité culturelle?}

L'étude du discours sur les compositeurs dans la presse du début du $\mathrm{XX}^{\mathrm{e}}$ siècle donne une idée assez précise de la représentation du monde de la création musicale à ce moment charnière de la modernité. Dans cette perspective, il est nécessaire d'identifier quels compositeurs sont considérés comme légitimes, mais aussi selon quels concepts (génie, vedette, artisan, etc.) cette légitimité est proposée, par qui et pour quel lectorat cible.

\section{La figure du génie et la rhétorique avant-courrière dans Le Mercure musical}

C'est le concept de génie - artiste inspiré dont l'œuvre sera un jour reconnue - qui est utilisé dans les pages du Mercure musical pour désigner les compositeurs légitimes. 
Laloy, par exemple, insiste sur l'inspiration naturelle de Debussy, par opposition au simple savoir-faire technique :

Il existe, et en nombre, d'honorables professeurs, directeurs ou anciens directeurs de conservatoires qui se sont illustrés aux concours par des fugues parfaites, et n'ont jamais pu écrire une seule ligne de musique réelle; c'est que pour satisfaire aux règles de ce genre il n'est pas besoin d'être musicien; il suffit de posséder un certain métier qui travaille de soi-même [...]. Mais ce qui sera toujours impossible, sauf aux authentiques enfants d'Harmonie, ce sera d'écrire valablement une musique libre, comme celle de Claude Debussy ; une musique qui se joue à sa guise [...]. Ce qu'il faudra ici, c'est autre chose que de la science, et plus que du goût ; c'est un instinct si délicat, une si sûre et profonde intuition, qu'on peut sans crainte parler de génie..$^{66}$

Dans une critique élogieuse des Miroirs de Ravel, Marnold aborde lui aussi la notion d'inspiration, mais s'attarde également à la réception des œuvres du compositeur. Il cite d'abord un ancien article dans le but de mettre en évidence la réalisation de la "prophétie » qu'il avait faite ( i il y a plus d'une année, j'écrivais dans le Mercure de France: "Il faut retenir le nom de Maurice Ravel: c'est celui d'un des maîtres de demain" »), avant d'affirmer que ceux qui ne reconnaissent pas la valeur de Ravel ne passeront pas à l'histoire : « Oublions-les, eux, leur acte et leurs noms introuvables déjà, la plupart, dans nos mémoires indifférentes ${ }^{67}$. Armande de Polignac utilise elle aussi la critique avant-courrière :

La très belle musique sera goûtée tôt ou tard, car, le temps aidant [,] le public s'élèvera jusqu'à elle, et s'il doit rester toujours un peu en retard, tant mieux pour les précurseurs de génie qui sont si vite détrônés. (Il s'agit ici, bien entendu, du public musicien, susceptible d'être impressionné par l'art vrai, et par l'art vrai seulement.) ${ }^{68}$

Ces trois exemples témoignent d'un discours qui porte sur les compositeurs jugés légitimes (ici Debussy et Ravel) mais qui parle aussi des autres - les illégitimes - et des critiques eux-mêmes qui se légitiment par association. La critique avant-courrière est au cœur de la stratégie argumentative du Mercure. Elle implique de dénigrer «les autres ", qu'il s'agisse de compositeurs, d'institutions, de critiques ou du public. Pour ce faire, l'ironie et le mépris sont les meilleures armes, et Marnold et Vuillermoz offrent les plus féroces attaques de ce type. Vincent d'Indy et la Schola cantorum sont l'ennemi numéro 1. Vuillermoz signe entre autres deux textes d'anticipation hautement sarcastiques où débattent des commissaires responsables d'un «dictionnaire biographique et critique des musiciens français des $\mathrm{XIX}^{\mathrm{e}}, \mathrm{XX}^{\mathrm{e}}$ et $\mathrm{XXI}^{\mathrm{e}}$ siècles ", éreintant Berlioz dans le premier et d'Indy dans le second. Le premier commissaire affirme d'emblée que la postérité n'a pas retenu les deux compositeurs :

Nos recherches habituelles de biographie, bibliographie et iconographie tendaient aujourd'hui à exhumer un nommé DINDY (Vincent) ou - pour respecter la naïve orthographe nobiliaire de nos aïeux, - d'INDY, compositeur de musique qui vécut vers la fin $d u X X^{e}$ siècle et le commencement $d u X X^{e}$. Vous remarquerez, Messieurs, que ce nom, pas plus que celui de Berlioz, n'a su résister à l'épreuve du temps. ${ }^{69}$

Les plumes se tournent aussi contre l'Académie des beaux-arts dans la foulée du scandale du prix de Rome : non seulement Ravel ne sort pas vainqueur du concours de 1905 (qui constituait alors sa cinquième tentative et dernière chance, en raison de l'âge, d'obtenir le fameux prix), mais il ne passe pas le premier tour alors qu'il avait été finaliste lors de précédentes éditions. La presse et les rubriques musicales s'emparent du sujet. Le Mercure, et plus particulièrement Marnold, attaque avec virulence la 
compétence des juges dans deux articles intitulés « Le scandale du Prix de Rome ${ }^{70}$. Le scandale mène à la démission de l'académicien et directeur du Conservatoire, Théodore Dubois, remplacé par Gabriel Fauré. Dans la section «Échos ", Marnold se réjouit de cette nomination tout en continuant à s'acharner sur l'ancienne administration :

M. Gabriel Fauré vient d'être nommé Directeur du Conservatoire. Un grand artiste remplace une honorable nullité à la barbe d'un prétentieux jocrisse. Le singulier verdict du tout récent " concours préparatoire ", n'est évidemment pas étranger au bienfait de cet heureux dénouement. Ainsi Gribouille crachait en l'air, sans se douter que ça lui retomberait sur le nez. ${ }^{71}$ ou sa personne ne font pas l'objet d'articles développés. Le Mercure consacre encore moins d'attention à Jules Massenet et Camille Saint-Saëns, pourtant deux des compositeurs les plus renommés au début du siècle, mis à part quelques flèches : Laloy juge les mélodies de Massenet «étirées et sucrées, qu'on croirait faites en guimauve, plates harmonies, sonorités aisées, mais creuses et monotones ${ }^{72}$, et qualifie l'opéra $L a$ Princesse jaune de Saint-Saëns d'œuvre «fanée » à l'« exotisme de convention » ${ }^{73}$.

arcasme du Mercure se tourne aussi contre d'autres publications ou musicographes. La jeune revue s'acharne particulièrement sur Auguste Mangeot du Monde musical, en raison, semble-t-il, d'un désaccord à propos d'Alfred Bruneau ${ }^{74}$. Mangeot se retrouve régulièrement cité dans le sottisier du Mercure, qui se défend d'être trop dur envers le critique, en poussant l'irrévérence encore un peu plus loin :

Plusieurs de nos lecteurs ont pu nous croire trop sévères pour un certain M. Mangeot ; non certes qu'ils le connussent, car alors, oh! alors... Mais enfin on n'aime pas à croire un homme doué de qualités intellectuelles aussi exactement négatives que le disaient nos aphorismes. Cela nous choque, nous contriste et nous humilie, comme le spectacle d'une trop grande misère. ${ }^{75}$

En septembre 1905, les «Échos» du Mercure publient un acrostiche dont les 14 vers forment « Mangeot est bête $»^{76}$.

Dans leurs textes engagés (qui côtoient plusieurs études musicologiques qui ne prennent pas part aux débats), les principaux collaborateurs du Mercure musical militent pour la musique de Debussy et de la jeune avant-garde gravitant autour de Ravel en recourant au concept de génie. Si Massenet et Saint-Saëns, qui occupent une place centrale dans Musica, sont parfois raillés dans Le Mercure, les principaux polémistes du périodique réservent leur énergie combative pour discréditer ce qu'ils considèrent être leurs réels ennemis: l'incompétence de plusieurs critiques, l'académisme de certaines institutions comme l'Académie des beaux-arts et le conservatisme de la Schola cantorum. Ainsi, la dynamique du Mercure rappelle celle des "petites revues " littéraires de la fin du XIX ${ }^{e}$ siècle " où la blague, l'attaque violente, l'insulte, le mépris tiennent lieu de rhétorique pour mettre en cause les adversaires; et où l'éloge démesuré, l'hagiographie et les annonces de génie à venir assurent une réclame fort peu subtile des alliés ${ }^{77}$.

\section{Le vedettariat et l'« esthétique de l'instantané » de Musica}

La quête de confrontation du Mercure contraste avec l'éclectisme consensuel retrouvé dans les pages de Musica qui est sans cesse réaffirmé : « nous ne bataillerons point. [...] un magazine illustré comme le nôtre se doit-il d'être paisible. Son rôle n'est pas de contraindre l'humanité, mais seulement de la distraire en l'instruisant $»^{78}$. Le magazine 
ne fait d'ailleurs aucune mention du scandale du prix de Rome de 1905, et se contente de présenter les vainqueurs du second tour ${ }^{79}$.

Les articles de Musica relèvent souvent de la réclame, et le ton employé pour parler des musiciens est presque toujours positif, bien que cela soit moins systématique à partir de 1910 (ce qui est certainement dû à l'arrivée de Leroux). Les questions esthétiques sont évacuées au profit d'un discours qui réaffirme sans cesse la grandeur des vedettes présentées. Les artistes qui ne sont pas considérés comme dignes de figurer au panthéon des grands sont généralement tout simplement absents des pages de Musica plutôt que d'être attaqués comme dans Le Mercure. La figure du génie est à l'occasion invoquée pour des compositeurs incontournables du XIX ${ }^{\mathrm{e}}$ siècle tels que Beethoven, Berlioz et Wagner, que tous et toutes dans la petite bourgeoisie peuvent reconnaître et qui ne peuvent être médiatisés en tant que vedettes pour la simple et bonne raison qu'ils ne sont plus vivants. Le magazine se garde bien cependant de trop utiliser une rhétorique avant-courrière pour les musiciens contemporains, une stratégie argumentative tournée vers le futur qui est peu compatible avec son «esthétique de l'instantané $»^{80}$.

Musica mise avant tout sur le présent, avec une ouverture vers les maitres du passé qui demeure restreinte et consensuelle quoique présentant aussi à l'occasion des figures moins connues de la Renaissance dans des textes de vulgarisation (qui demeurent cependant loin des études musicologiques publiées à cette époque, par exemple dans la Revue d'histoire et de critique musicales ou même dans Le Mercure musical). Chaque numéro comporte une couverture couleur et une page de titre, toutes deux présentant une photographie grand format d'un ou plusieurs artistes.

III. 3. Mary Garden et Isidore de Lara, respectivement sur la couverture et page de titre du numéro de février 1904 de Musica.
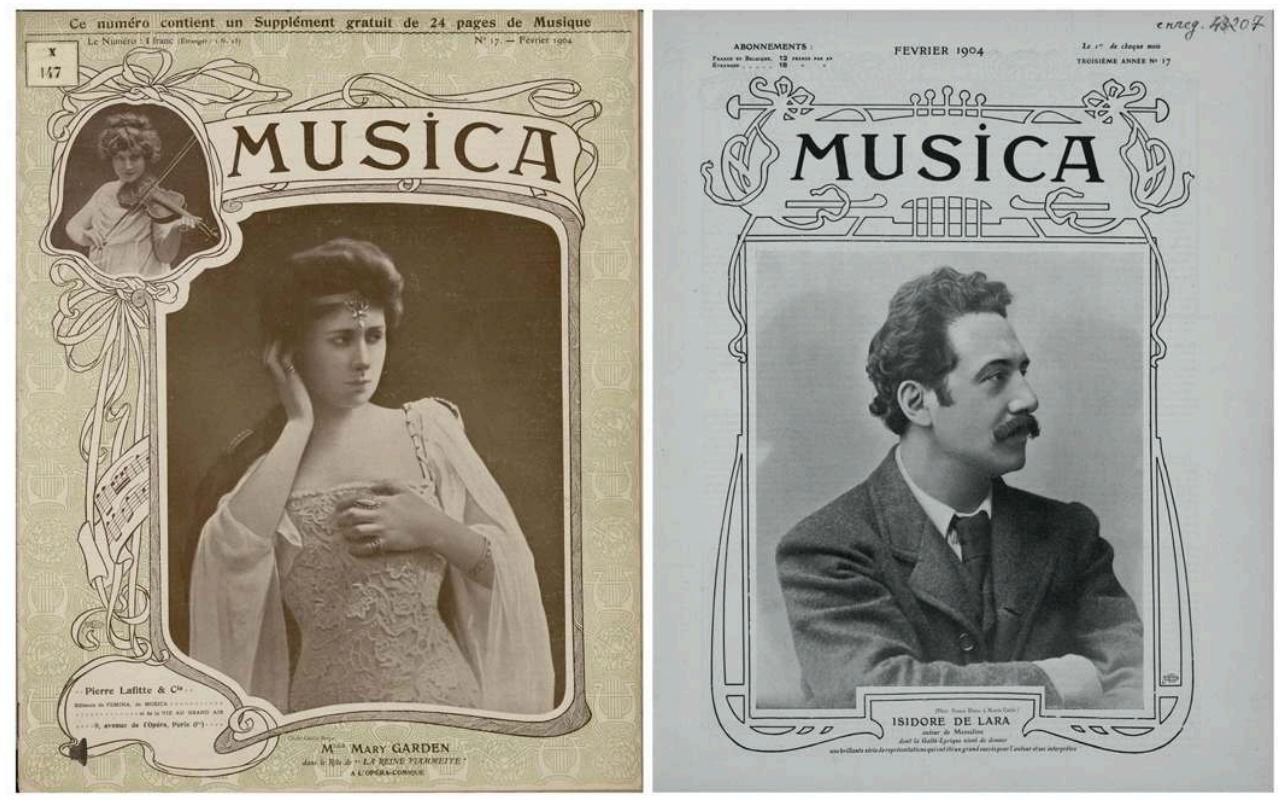

Dans $75 \%$ des cas, cette couverture et cette page de titre proposent un portrait de personnalités vivantes, contre $17 \%$ pour des personnalités décédées avant la fondation du magazine en 1902. Seulement 7\% ne présentent pas de personnalités identifiables, et $1 \%$ sont consacrées à des figures récemment décédées à qui elles rendent hommage. 
Graph. 1. Pourcentage de couvertures de Musica présentant des personnalités vivantes ou décédées.

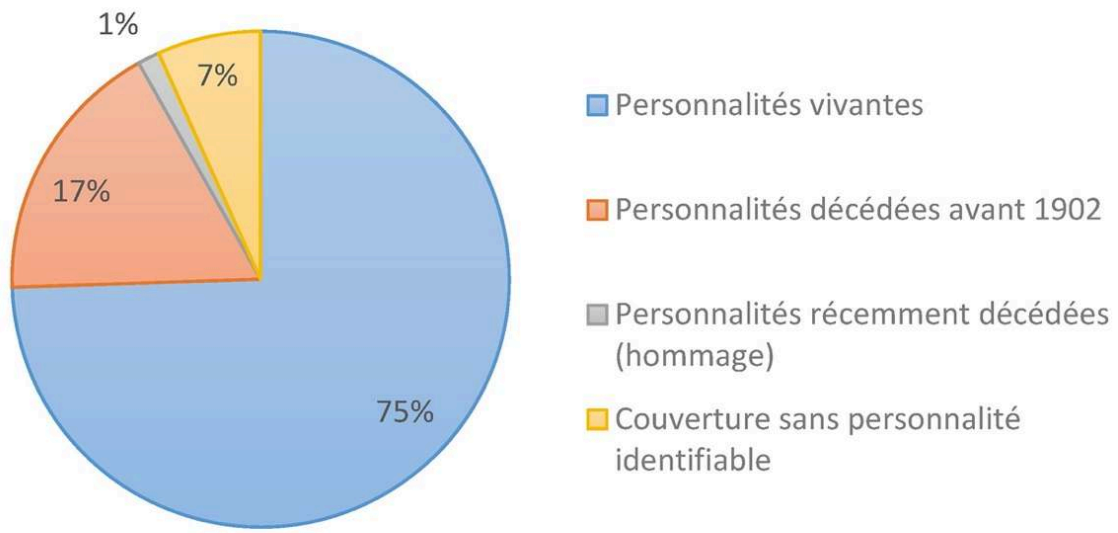

$39 \%$ des couvertures et pages de titre sont consacrées à des cantatrices (28\%) et chanteurs (11\%) associés à la scène opératique, généralement seuls et bien mis en valeur dans le costume de leur plus récent rôle. Une personnalité pratiquant la composition est présente sur $42 \%$ d'entre elles.

Graph. 2. Fonctions des personnalités figurant sur les couvertures de Musica.

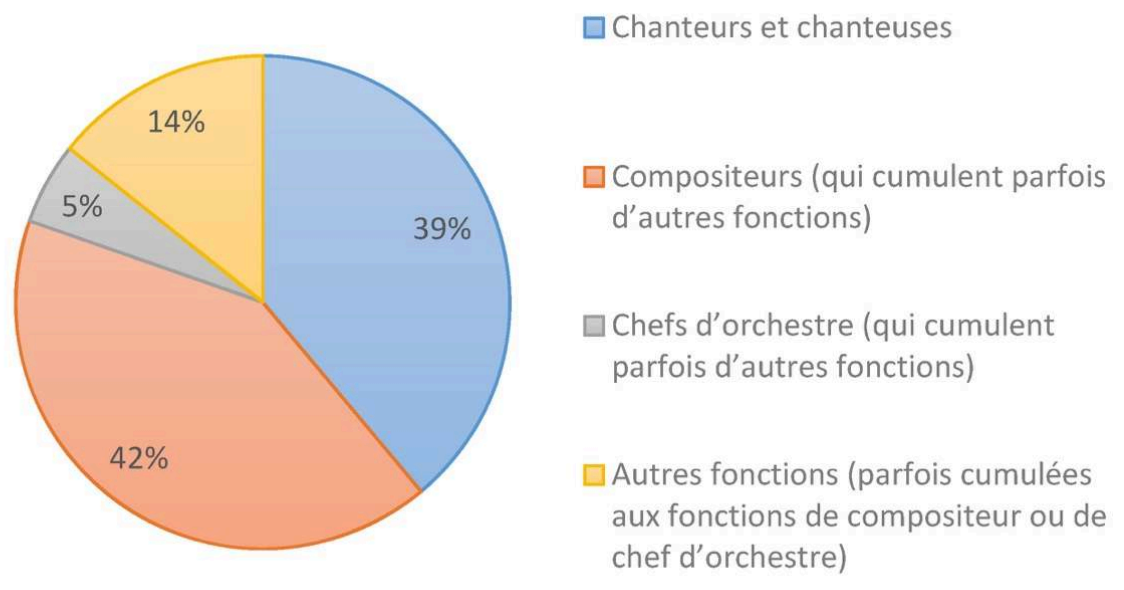

Ce chiffre est considérable et s'explique en partie par le fréquent cumul de fonctions pour les compositeurs qui sont souvent aussi chefs d'orchestre, administrateurs ou professeurs. C'est parfois en raison d'une nomination à la tête d'un orchestre ou d'une institution et non pour leur activité compositionnelle que certains compositeurs figurent en couverture ou page de titre. Malgré cette nuance, et si l'on considère qu'en " régime médiatique ", la célébrité est surtout conférée " aux artistes interprètes - et non plus créateurs, comme au XIX ${ }^{\mathrm{e}}$ siècle » (ces derniers érigeront d'ailleurs souvent la visibilité en antivaleur) ${ }^{81}$, la visibilité accordée aux compositeurs est étonnamment élevée dans Musica. C'est que le magazine voit le jour au début de l'ère de la visibilité, alors que la figure du créateur est encore imposante. Aussi, plusieurs compositeurs 
jouent volontiers le jeu du vedettariat, à une époque où le coût de la visibilité en termes de perte de crédibilité aux yeux des pairs ne se posait pas aussi clairement que par la suite. La place du musicien-créateur dans ce nouvel écosystème médiatique qu'est le magazine est encore en cours de définition.

Parmi les couvertures et pages de titre consacrées à des compositeurs, 57\% présentent des personnalités vivantes, $40 \%$ qui sont décédées avant 1902 et 3\% qui sont récemment décédées (en guise d'hommage). Mozart, Beethoven, Schumann, Berlioz, Liszt, Gounod, Wagner sont bien représentés. Il s'agit là du reflet du canon issu des XVIII et $\mathrm{XIX}^{\mathrm{e}}$ siècles qui se renforce sans cesse. Du côté des compositeurs contemporains, contrairement au Mercure musical qui cherche à révéler les artistes de la jeune avantgarde, Musica médiatise surtout ceux déjà très connus et qui se distinguent par leurs œuvres lyriques ou leurs fonctions institutionnelles: Jules Massenet (très actif sur la scène lyrique, professeur au Conservatoire et membre de l'Académie des beaux-arts), Camille Saint-Saëns (actif sur la scène lyrique et membre de l'Académie des beaux-arts), Théodore Dubois (membre de l'Académie des beaux-arts et directeur du Conservatoire jusqu'en 1905), Gabriel Fauré (professeur au Conservatoire dont il devient le directeur en 1905 et nommé membre de l'Académie des beaux-arts en 1909), Ernest Reyer (connu pour ses opéras et membre de l'Institut) et même Vincent d'Indy (directeur de la Schola cantorum) jouissent tous d'une visibilité considérable, par le biais de photographies en couverture ou en page de titre, de portraits (parfois en pleine page), de clichés de groupe, de caricatures ou encore par la publication d'articles ainsi que la diffusion de partitions dans l'Album-Musica. Parmi les autres compositeurs qui profitent d'une visibilité intéressante, mentionnons Richard Strauss, Ruggero Leoncavallo et Giacomo Puccini - trois non-Français -, Charles Lecocq - un contemporain de Saint-Saëns qui s'est illustré dans l'opérette -, Alexandre Georges, André Messager, Alfred Bruneau, Gustave Charpentier, Camille Erlanger et Xavier Leroux - tous nés dans les 1850 ou début des années 1860 -, ainsi que le plus jeune Reynaldo Hahn et éventuellement Raoul Laparra. Il s'agit tous de noms liés à la scène lyrique et/ou faisant partie du réseau d'Astruc. Contrairement à la profession de chanteur où les femmes sont plus visibles que les hommes, la fonction de compositrice n'est mise de l'avant qu'à de rares occasions $^{82}$.

Le milieu de l'opéra offre à la presse «un réservoir inépuisable de photos magnifiques $»^{83}$, mais ce n'est pas la seule raison pour laquelle les compositeurs lyriques sont les plus visibles dans les pages de Musica. En raison de la plus grande couverture journalistique des théâtres par rapport aux salles de concert, ces compositeurs peuvent plus aisément accumuler un « capital de visibilité » sur lequel le magazine de Lafitte mise pour accroître ses ventes. Le capital de visibilité « augmente par sa simple manifestation : plus une vedette est célèbre, plus son image est diffusée, et plus son image est diffusée, plus sa célébrité s'accroît $»^{84}$, ce qui a comme conséquence de sans cesse renforcer l'engouement envers les vedettes et, du même coup, celui envers les périodiques qui diffusent leur nom et leur image.

Dans la logique de Musica et autres magazines participant au "régime médiatique », la célébrité se justifie avant tout par la gloire préexistante (plutôt que par le talent ou l'originalité). Les chapeaux et légendes mettent l'accent sur la gloire de facto du musicien, ce qui justifie sa présence dans ses pages. Par exemple, le chapeau d'un article de novembre 1905 consacré aux auteurs de l'opéra Miarka aujourd'hui oublié, Alexandre Georges (compositeurs) et Jean Richepin (librettiste), affirme que leur 
« célébrité universelle et de bon aloi vaut bien qu'on en parle » ${ }^{85}$. Le numéro de juillet 1907 présente en couverture « le grand compositeur André Messager, le futur directeur de l'Opéra, auteur de Fortunio qui vient d'être joué à l'Opéra-Comique avec le plus vif et le plus légitime succès $\|^{86}$. Les légendes des illustrations font appel à des qualificatifs comme "succès ", " éminent ", "grand ", " acclamé ", « illustre ", etc. Ce système de légendes et chapeaux insistant sur la grandeur est unique à Musica et ne se retrouve pas, par exemple, dans Comcedia illustré, supplément bimensuel richement illustré du quotidien culturel Comœedia et seul réel concurrent du magazine de Lafitte à partir de 1908. Musica insiste aussi sur la grandeur des compositeurs lorsque ceux-ci prennent la parole dans le magazine (article, interview, réponse à une enquête), autorité souvent mise à profit pour légitimer le magazine lui-même et ses activités connexes, comme lorsqu'est rapporté, en janvier 1904, « ce que pensent MM. Massenet, Théodore Dubois et Ch. Lenepveu, membres de l'Institut, du Grand Tournoi International de Musica ». On lit à propos de Lenepveu, «le célèbre compositeur [...] connu pour être celui qui "fait" le plus de Prix de Rome ", qu'« il est ravi que Musica ait procuré à quelques-uns [des jeunes musiciens] les moyens de se faire connaître, leur ait facilité une édition prochaine $»^{87}$.

41 En plus d'élever la gloire préexistante comme une marque d'autorité, Musica utilise une autre stratégie dans sa mise en scène des grands compositeurs : l'exposition de la vie privée des artistes au regard curieux des lecteurs et lectrices qui découvrent en photo leur intimité. Les compositeurs, étonnamment davantage que les interprètes, sont présentés à la fois dans ce qu'ils ont de singulier - ce sont des vedettes - et d'ordinaire leur quotidien (vie de famille, travail, vacances, etc.) qui sous certains aspects n'est pas si éloigné de celui des lecteurs et lectrices de Musica, du moins dans l'esprit de ces derniers qui rêvent peut-être d'une vie idéale comme celle des artistes qui leur sont présentés. Dans l'article précédemment mentionné sur Alexandre Georges et Jean Richepin, on retrouve une photographie du compositeur avec ses deux jeunes filles et une du poète en famille. En septembre 1903, l'article "Nos musiciens aux champs » présente des photographies de compositeurs ou pianistes-compositeurs durant leurs vacances. On y voit notamment Saint-Saëns, Massenet, Reyer, Dubois à la chasse et Bruneau en barque. En novembre 1903, un article intitulé « Puccini \& la Tosca» montre le cabinet de travail du compositeur, reproduit un portrait de son grand-père, et met en scène Puccini, en compagnie de son chien favori, conduisant son automobile, revenant de la chasse ou encore sur son canot à essence. 
III. 4. « Puccini \& la Tosca » (Musica, novembre 1903).
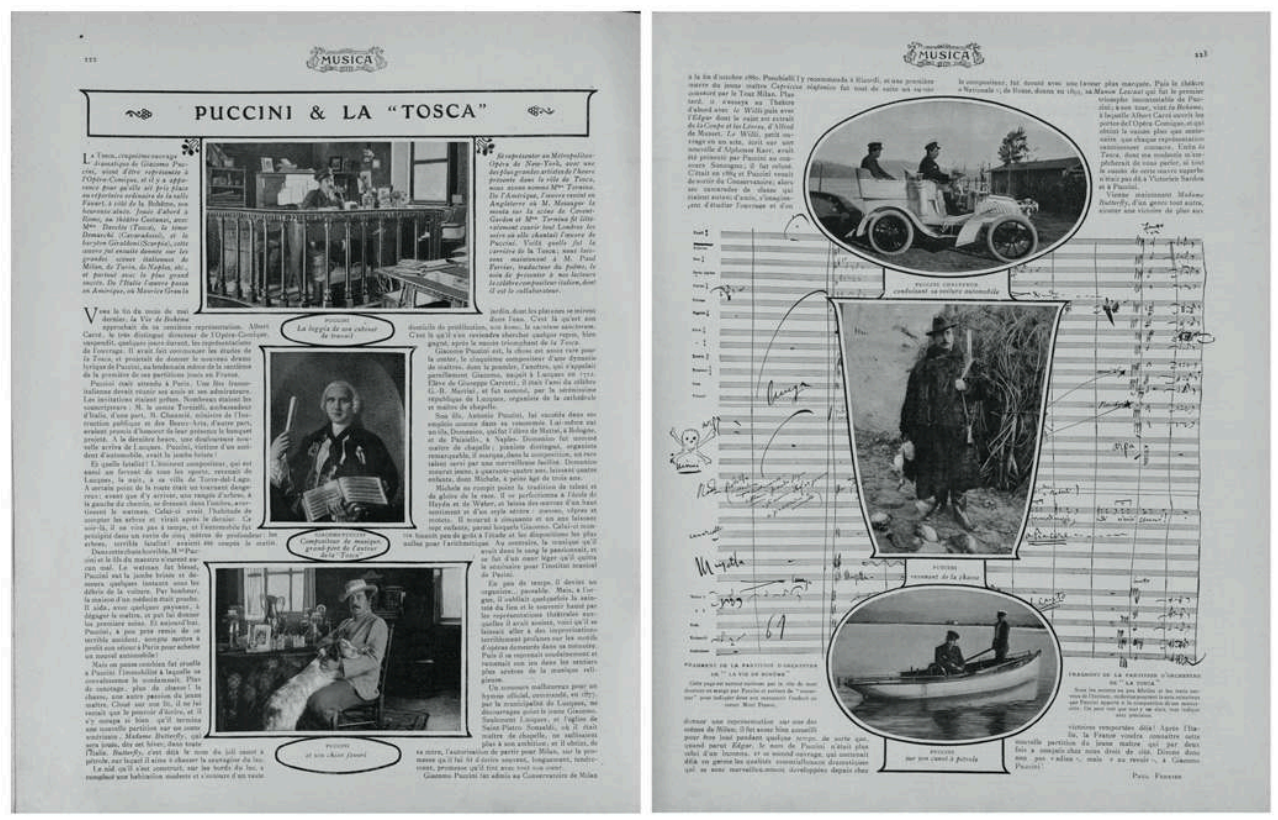

Cet article sur Puccini est également représentatif d'une autre stratégie qu'utilise Musica pour « rapprocher » les vedettes du lecteur ou de la lectrice : il est de la plume d'un proche ou collaborateur du compositeur, ici Paul Ferrier, traducteur de la Tosca.

C'est sur le désir (illusoire) de devenir soi-même une célébrité que joue Musica en présentant la grandeur culturelle comme un phénomène accessible aux gens ordinaires $^{88}$. En d'autres mots, Musica vend du rêve (tout en proposant une série de textes pédagogiques et de vulgarisation). Évidemment, le «rêve » ne se concrétise que très rarement, car bien que les noms et parfois même les photos des gagnants des concours (composition et interprétation) que Musica organise pour ses abonnés soient publiés, cette voie n'est pratiquement jamais professionnalisante et la visibilité acquise n'est qu'éphémère. La principale conséquence du processus d'identification à la vedette n'est pas que le lecteur (ou la lectrice) accède lui-même à la célébrité ; plutôt, ce dernier développe généralement un sentiment d'attachement qui l'encourage à consommer voire à collectionner - des effigies et des informations sur les vedettes auxquelles il s'identifie ou qu'il souhaite " posséder ». Ce rapport émotif aux célébrités qu'encourage Musica a ainsi un potentiel très lucratif pour le magazine. L'image des musiciensvedettes est même utilisée à quelques occasions dans un cadre publicitaire, comme lorsqu'une photographie du compositeur Xavier Leroux avec autographe sert à vanter le porteplume réservoir de marque Onoto. 


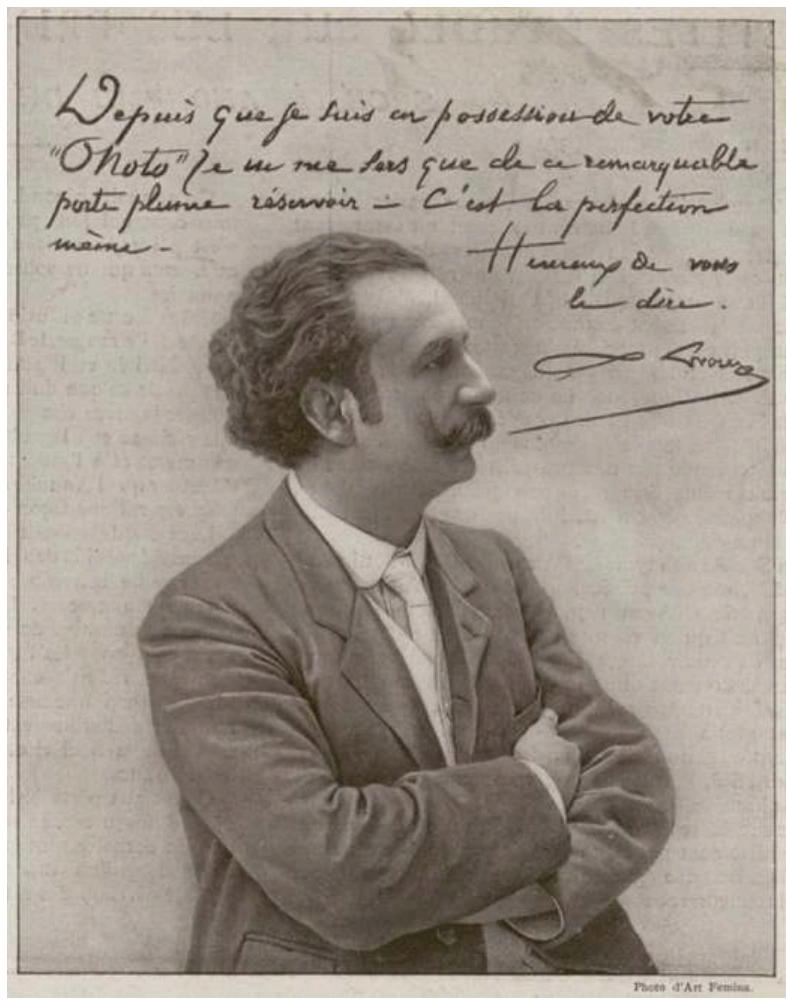

Soulignons que Debussy n'est que peu présent dans les pages de Musica, ce qui peut surprendre considérant l'importance médiatique de Pelléas et Mélisande en 1902, opéra autour duquel les Apaches avaient organisé un «bataillon sacré »" ${ }^{89}$. Ce n'est qu'à partir de 1907 (voire 1908) que Musica donne un peu plus de visibilité à Debussy et ses œuvres. Debussy semble ne pas s'être prêté au jeu médiatique ${ }^{90}$, à l'instar d'un Mallarmé. Le vedettariat ne dépend effectivement pas uniquement du magazine qui le rend possible, et une collaboration de la part des potentielles vedettes est nécessaire.

À partir de 1910 et plus encore de 1911, les compositeurs de la jeune «avant-garde profondément influencés par le symbolisme et un tant soit peu dandys $»^{91}$ qui avaient été défendus dans Le Mercure en 1905-1906 commencent à obtenir une plus grande visibilité dans Musica, dans des proportions qui demeurent somme toute modestes, alors qu'ils en étaient jusqu'alors presque totalement absents. Déodat de Séverac va jusqu'à figurer sur la page de titre du numéro de décembre 1911. Cela est en partie dû à l'arrivée de Xavier Leroux au poste de rédacteur en chef en novembre 1910. Celui-ci modifie l'orientation éditoriale du magazine en laissant une plus grande place aux chroniques et comptes rendus de concerts ( 2 à 4 pages qui sont cependant moins riches en iconographie que le reste du numéro) où se côtoient plusieurs points de vue. Le ton parfois plus sévère ou partisan qu'on y trouve contraste avec celui toujours élogieux des pages illustrées destinées à être feuilletées. Par exemple, la légende de la page illustrée consacrée à L'Heure espagnole de Ravel est très positive et insiste sur le succès de l'œuvre ( le brillant petit acte de M. Franc-Nohain, pour lequel M. Maurice Ravel écrivit une partition du plus curieux intérêt, peut se prévaloir justement d'une interprétation hors de pair. [...] [Les interprètes] contribuèrent certes pour beaucoup à la réussite de cet ouvrage très applaudi »), tandis que Leroux, qui se montre à l'occasion assez sévère envers les compositeurs de l'avant-garde dans sa rubrique « La musique au 
théâtre ", manifeste vivement son désaccord avec la démarche de Ravel et son statut d'étoile montante de la musique moderne :

Chef d'école, il ne l'est pas, ne peut l'être ; son art, si volontaire qu'il soit, n'étant, quant à présent, ni assez définitif, ni assez personnel, pour valoir à son auteur une telle épithète. Tout au plus ses essais peuvent-ils, pour le moment, servir de modèles - de très mauvais modèles au demeurant - à de maladroits plagiaires [...] et nous pouvons prévoir d'ores et déjà la genèse d'œuvres nulles, mauvaises, détestables... [...] M. Maurice Ravel a, comme quiconque, son violon d'Ingres. Le sien est de se croire un humoriste. [...] Pourquoi, au lieu de rire, de s'esclaffer comme le lui demandait souvent son sujet, n'a-t-il que ricané, ricané comme ces jeunes vieux qui s'interdisent le rire franchement gai, dispensateur de santé ? Pourquoi toute chose se présente-t-elle à nous avec un aspect aussi rabougri ?...92

Dans un souci d'éclectisme des points de vue, Leroux confie une rubrique régulière des "chroniques et critiques de Musica» à Vuillermoz, aux opinions généralement opposées aux siennes, qui continue à mettre sa plume combative, certes un peu moins mordante que dans Le Mercure musical, au service de l'avant-garde. Cette collaboration régulière entre Musica et Vuillermoz survient quelques mois à peine après la publication en page de titre de la fameuse photographie de la Société musicale indépendante, nouvellement fondée : on y voit plusieurs des membres fondateurs (dont Ravel et Vuillermoz) disposés autour de Fauré, son président, assis au piano.

III. 6. « Une lecture à la Société musicale indépendante » (Musica, juin 1910).

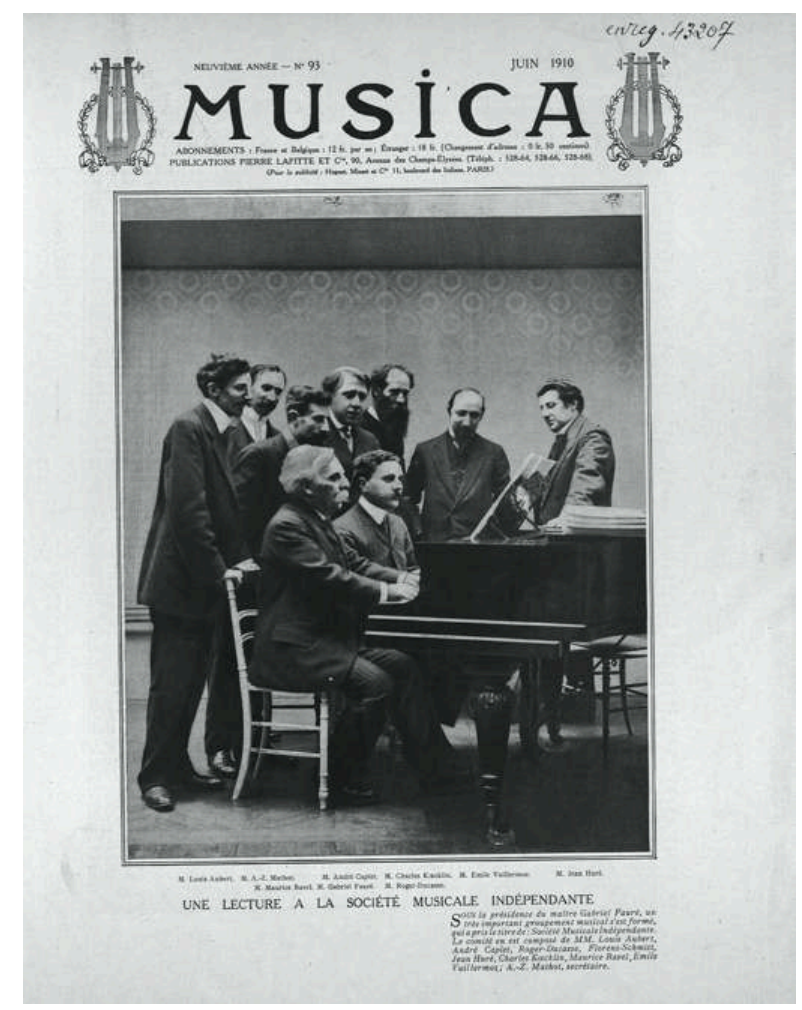

Le magazine de Lafitte se transforme sensiblement à partir de 1910 en donnant une plus grande visibilité à la jeune avant-garde, mais c'est aussi l'avant-garde qui a changé - elle s'est imposée, notamment grâce aux combats menés dans d'autres périodiques que Musica tels que Le Mercure musical - et ses porte-voix, comme Vuillermoz, ont adapté leurs stratégies en investissant aussi la presse à plus large tirage tel que le magazine de Lafitte. Une certaine image de l'avant-garde est aussi en vogue durant ces 
années en raison du succès des Ballets russes à Paris, dont Astruc était d'ailleurs le promoteur. Le bimensuel Comœdia illustré, qui s'adresse davantage à l'élite de la société que Musica, joue pour beaucoup dans cette «mise à la mode» de l'avant-garde ${ }^{93}$ qui percole dans Musica bien que les deux périodiques occupent des créneaux différents.

\section{Conclusion}

Musica et Le Mercure musical ont en commun de servir de puissant outil promotionnel pour les compositeurs. Musica met à profit sa formule magazine pour promouvoir des créateurs déjà connus en raison de leurs œuvres lyriques ou de leurs fonctions institutionnelles. Ces derniers sont médiatisés comme vedettes, ce qui renforce leur légitimité auprès du lectorat de Musica. L'engouement envers certains artistes qu'entretient le magazine se révèle un atout dans la carrière des compositeurs qui se prêtent au jeu, tout en représentant un potentiel lucratif pour Lafitte et Astruc. À l'inverse, Le Mercure musical, qui revendique une haute légitimité culturelle, propose un panthéon alternatif non seulement à celui de Musica, mais aussi à celui d'autres périodiques comme la Revue d'histoire et de critique musicales. Cette dernière, qui présente des affinités avec la Schola cantorum, recourt davantage au concept d'artisan pour désigner les grands compositeurs et rejette à la fois le vedettariat et la rhétorique avant-courrière.

Musica et Le Mercure musical de même que la Revue d'histoire et de critique musicales que nous venons de mentionner ne balisent pas à eux seuls le monde musical du début du $\mathrm{XX}^{\mathrm{e}}$ siècle, dont les dynamiques complexes reposent sur plusieurs autres paramètres que la seule conception de la grandeur culturelle. Cela étant dit, la comparaison du Mercure et de Musica permet de mettre en relief la façon dont les spécificités éditoriales et les nécessités des différentes sphères de sociabilités des périodiques (rentabilité, légitimation culturelle, etc.) se reflètent dans la façon de promouvoir les artistes et notamment les compositeurs. Bien qu'elles soient antagonistes à bien des égards, des ponts existent entre les deux publications, et plus particulièrement lorsque Musica octroie une plus grande visibilité (qui reste somme toute restreinte) à l'avant-garde auparavant défendue dans Le Mercure, et qu'une tribune régulière est confiée à Vuillermoz. Musica semble d'ailleurs avoir servi de tremplin dans la carrière musicographique de ce dernier, en mettant fin à quelques années d'instabilité dans ses collaborations avec différents organes de presse : peu de temps après Musica, il entre en 1911 comme critique à la Revue musicale SIM (dont il deviendra, nous l'avons mentionné, le rédacteur en chef en 1912) puis en 1913 à Comodia. S'ouvriront ensuite à lui les portes de plusieurs quotidiens et hebdomadaires prestigieux ou à tirage important, comme Le Temps ${ }^{94}$, Excelsior, L'Illustration et Candide, où il continue à promouvoir sensiblement les mêmes idées esthétiques qui ont cependant perdu leur caractère révolutionnaire.

La comparaison entre Musica et Le Mercure musical fait ressortir comment ces périodiques et le discours qu'ils portent s'inscrivent (ou non) dans la durée. L'histoire de la musique semble reprendre à bien des égards une narration mettant de l'avant les pionniers - c'est-à-dire les génies qui seront un jour reconnus tels qu'ils ont été promus dans leurs débuts dans Le Mercure musical - plutôt que les compositeurs jouissant d'un succès et qui ont été médiatisés comme vedettes, notamment dans Musica. La " petite revue » musicale qu'est Le Mercure musical aurait-elle agi, à l'instar de ses consœurs 
littéraires, comme une "fabrique médiatique de la postérité »" ensuite relayée par la grande presse? Le statut des artistes est intimement lié à un système de valeurs qui varie selon les périodiques. L'étude comparée de publications aux lignes éditoriales différentes permet de nuancer l'axiologie véhiculée par l'historiographie de la musique française, qui présente les premières années du siècle essentiellement comme celles de Debussy et de Ravel.

\section{NOTES}

1. La Vie au Grand Air appartient d'abord à l'éditeur Jules Rueff et $C^{\text {ie }}$ qui confie à Lafitte la rédaction. Lafitte fait partie du groupe d'actionnaires qui achètent la revue en 1900, et il en devient aussitôt le directeur (Thierry Gervais, L'illustration photographique: Naissance du spectacle de l'information (1843-1914), thèse de doctorat, Paris, EHESS, 2007, p. 408).

2. Thierry Gervais, «La presse illustrée : 2. Les premiers magazines illustrés, de la gravure à la photographie (1898-1914)", dans Dominique Kalifa, Philippe Régnier, Marie-Ève Thérenty et Alain Vaillant (dir.), La civilisation du journal : Histoire culturelle et littéraire de la presse française au XIX ${ }^{e}$ siècle, Paris, Nouveau monde éditions, 2011, pp. 453-463.

3. Ibid., pp. 455-457.

4. Sur l'admiration que suscitaient les castrats, voir Martha Feldman, The Castrato : Reflections on Natures and Kinds, Berkeley, University of California Press, 2015. Sur la célébrité des virtuoses au $\mathrm{XIX}^{\mathrm{e}}$ siècle et plus particulièrement le cas de Franz Liszt, voir Antoine Lilti, Figures publiques: L'invention de la célébrité, 1750-1850, Paris, Fayard, 2014, pp.316-326. La série de portraits de chanteurs et chanteuses qu'Arthur Pougin a publiée entre 1868 et 1874 dans Le Ménestrel constitue un autre exemple éloquent d'utilisation du concept de vedette au XIX ${ }^{e}$ siècle.

5. Nathalie Heinich, L'élite artiste: Excellence et singularité en régime démocratique et De la visibilité : Excellence et singularité en régime médiatique, Paris, Gallimard, 2005 et 2012.

6. Sur les origines de la célébrité et la révolution médiatique, voir Lilti, Figures publiques: L'invention de la célébrité, 1750-1850.

7. Heinich, De la visibilité, pp. 43-52.

8. Lilti, Figures publiques: L'invention de la célébrité, 1750-1850, p. 76.

9. Michel Duchesneau, "Composer avec l'image: Les compositeurs dans Musica (1902-1914) », dans Laurence Brogniez, Clément Dessy et Clara Sadoun-Édouard (dir.), L'artiste en revues: Arts et discours en mode périodique, Rennes, Presses universitaires de Rennes, 2019, pp. 375-397.

10. Juliette Dugal, « Pierre Lafitte, "le César du papier couché” », dans Jean-Luc Buard (dir.), Les Éditions Pierre Lafitte [I], numéro thématique de Le Rocambole: Bulletin des amis du roman populaire, $\mathrm{n}^{\circ} 10,2000$, pp. 13-38: 18. 
11. Pierre Albert, « La presse française de 1871 à 1940 », dans Claude Bellanger, Jacques Godechot, Pierre Guiral et Fernand Terrou (dir.), Histoire générale de la presse française, tome III : De 1871 à 1940, Paris, Presses universitaires de France, 1972, pp. 133-622 : 280.

12. Gabriel Astruc, Le Pavillon des fantômes: Souvenirs, nouvelle édition augmentée présentée par Olivier Corpet, Paris, Mémoire du livre, 2003, p. 342. Le projet initial portait le nom de La Vie musicale (Cesar A. Leal, Re-Thinking Paris at the Fin-De-Siècle: A New Vision of Parisian Musical Culture from the Perspective of Gabriel Astruc (1854-1938), thèse de doctorat, Lexington, University of Kentucky, 2014, pp. 63-67).

13. Astruc signe quelques articles pour Musica avec ce pseudonyme.

14. Astruc, Le Pavillon des fantômes, pp. 113-114 et 297.

15. Ibid., p. 341. C'est nous qui soulignons.

16. Leal, Re-Thinking Paris at the Fin-De-Siècle, pp. 109-163 et 228-275.

17. Camondo et Deutsch de la Meurthe avaient tous les deux reçu leur éducation juive du père d'Astruc, un important rabbin (Astruc, Le Pavillon des fantômes, pp. 49-50).

18. Leal, Re-Thinking Paris at the Fin-De-Siècle, pp. 101-109.

19. Astruc, Le Pavillon des fantômes., p. 344. N'ayant pas les détails du montage financier de Musica, nous ne sommes pas en mesure de déterminer la part de ce financement.

20. Sur les spécificités de chaque rédaction, voir Carla E. Biberdorf, Musica: An Early Twentieth-Century French Music Journal, mémoire de maîtrise, Vancouver, University of British Columbia, 1984, pp. 18-33.

21. Astruc, Le Pavillon des fantômes., p. 344.

22. Un "pendentif Musica», un "dessus de clavier Musica» ou un "rouleau portemusique Musica» (Duchesneau, «Composer avec l'image », p. 378).

23. Astruc, Le Pavillon des fantômes, p. 342.

24. Duchesneau, « Composer avec l'image », p. 378.

25. «Notre salle d'exposition », Musica, 4, janvier 1903, p. 64.

26. Sur cette salle, voir l'entrée "Femina, théâtre » dans Nicole Wild, Dictionnaire des théatres parisiens, 1807-1914, Lyon, Symétrie/Venise, Palazzetto Bru Zane, 2012, pp. 142-143.

27. Pierre Lafitte et $\mathrm{C}^{\mathrm{ie}}$, « Notes des éditeurs », Musica, 53, février 1907, p. 32.

28. Astruc, Le Pavillon des fantômes, p. 345.

29. Musica, « À nos abonnés », Musica, 114, mars 1912, p. 48.

30. Musica, «Un conservatoire d'amateurs créé par Femina et Musica », Musica, 61, octobre 1907, p. 160.

31. Astruc, Le Pavillon des fantômes, p. 343.

32. Sur cet aspect, le magazine musical de Lafitte se démarque de Femina qui s'adresse plutôt à la bourgeoisie aisée (Christophe Charle, Le siècle de la presse (1830-1930), Paris, Seuil, 2004, p. 193).

33. Musica, «L'anniversaire de Musica », Musica, 14, novembre 1903.

34. Astruc, Le Pavillon des fantômes, p. 343.

35. Anne-Claude Ambroise-Rendu, « Les faits divers », dans Kalifa, Régnier, Thérenty et Vaillant (dir.), La civilisation du journal, pp. 979-997 : 996.

36. «La princesse Louise de Saxe compositeur », Musica, 6, mars 1903, p. 92.

37. Note précédant Charles Malherbe, "Maria Daurignac, compositeur ", Musica, 3, décembre 1902, p. 46.

38. «Concours de Musica», Musica, 7, avril 1903, p. 113.

39. Musica, "Cours d'enseignement musical», Musica, 1, octobre 1902, p.2. Trois sections composent cette rubrique : "L'enseignement de la musique par la mère de famille », «Pour 
apprendre à chanter : Conseils et exercices pratiques » et une autre consacrée à l'enseignement du piano.

40. Charle, Le siècle de la presse, p. 189.

41. L'expression est de Laloy (La musique retrouvée, 1902-1927 [1928], Paris, Desclée de Brouwer, 1974, p. 138). Une réduction était offerte aux abonnés du Mercure de France (10 francs pour l'abonnement annuel plutôt que 12).

42. Pour un aperçu du phénomène des petites revues, voir Yoan Vérilhac, "La petite revue ", dans Kalifa, Régnier, Thérenty et Vaillant (dir.), La civilisation du journal, pp. 359-373.

43. Laloy, La musique retrouvée, p. 138. Pour une généalogie du Mercure musical, voir Michel Duchesneau, «French Musicology and the Musical Press (1900-14) : The Case of La Revue musicale, Le Mercure musical and La Revue musicale SIM », Journal of the Royal Musical Association, 140, 2, 2015, pp. 243-272.

44. Une attention particulière est réservée à la musique « orientale » (de l'Algérie au Cambodge) qui ne se retrouve pas dans les autres revues accueillant la musicologie alors en cours d'institutionnalisation, comme la Revue d'histoire et de critique musicales. Cela est probablement dû aux intérêts et réseaux de Louis Laloy qui est autant spécialiste de l'histoire de la musique de la Grèce antique, de Chine ou du Cambodge que de Rameau et de Moussorgski. En plus d'être critique et musicologue, il est actif comme auteur et comme traducteur (il maîtrise sept langues outre le français). Il a aussi étudié la composition. (Michel Duchesneau, Martin Guerpin et MariePier Leduc, «Musicologie et presse musicale en France (1889-1914) », Un siècle de musicologie en France, vol. 1: Histoire intellectuelle de la Revue de musicologie, numéro spécial de la Revue de musicologie, 103, 2, 2017, pp. 71-116: 105-107).

45. Pascal Lécroart, «Entre musique et littérature : L'inventivité formelle et rhétorique dans Le Mercure musical », dans Timothée Picard (dir.), La critique musicale au XXe siècle, Rennes, Presses universitaires de Rennes, 2020, pp. 679-690:679 et 682.

46. Entre 1905 et 1906, Laloy rédige huit textes pour la section principale de revue et 54 pour la « revue de la quinzaine », tandis que Marnold en publie respectivement neuf et huit.

47. Jann Pasler, "A Sociology of the Apaches: "Sacred Battalion" for Pelléas", dans Barbara L. Kelly et Kerry Murphy (dir.), Berlioz and Debussy: Sources, Contexts and Legacies, Burlington, Ashgate, 2007, pp. 149-166; Malou Haine, "Cipa Godebski et les Apaches », Revue belge de musicologie/Belgisch Tijdschrift voor Muziekwetenschap, 60, 2006, pp. 221-266.

48. Du moins durant ces années. Laloy et Marnold se disputeront tous les deux avec certains membres des Apaches (notamment Vuillermoz) dans les années suivantes.

49. Pan, «Échos: Concours de Rome », Le Mercure musical, 1, 1, 15 mai 1905, p. 46.

50. Pan, «Échos : M. Maurice Ravel », Le Mercure musical, 1, 12, $1^{\mathrm{er}}$ novembre 1905, p. 501.

51. Maurice Delage, «Les premiers amis de Ravel », dans Colette et al., Maurice Ravel par quelquesuns de ses familiers, Paris, Éditions du tambourinaire, 1939, pp. 97-113 : 98.

52. Lettre de Maurice Ravel à Émile Vuillermoz, 22 avril 1910, Harry Ransom Center, Austin (TX), Carlton Lake Collection, boîte 203, dossier 12, repris dans Maurice Ravel, L'intégrale: Correspondance (1895-1937), écrits et entretiens, édition établie, présentée et annotée par Manuel Cornejo, Paris, Le Passeur Éditeur, 2018, p. 231.

53. Félix Gaiffe, «Émile Vuillermoz : Écrivain et compositeur », Franche-Comté et Monts Jura: Revue régionale mensuelle, 5, 51, octobre 1923, pp. 46-48: 47.

54. Maurice Ravel, « Noël des jouets », Le Mercure musical, 2, 10, 15 mai 1906, p. 454.

55. Léon Vallas, Vincent d'Indy, vol. 2, La maturité, la vieillesse (1886-1931), Paris, Albin Michel, 1950, p. 61.

56. Voir Michel Duchesneau, L'avant-garde musicale à Paris de 1871 à 1939, Liège, Mardaga, 1997, pp. 65-92.

57. Laloy, La musique retrouvée, p. 138. 
58. À la suite de quoi Ravel signe une entente avec la plus prestigieuse maison Durand (Roger Nichols, Ravel, New Haven, Yale University Press, 2011, p. 79). Astruc a lui aussi publié des œuvres de Ravel, le Quatuor pour instruments à cordes et Shéhérazade, toutes les deux en 1904.

59. Le Mercure offre une remise de $40 \%$ sur ces partitions à la suite d'une entente avec la maison Leduc.

60. Émile Vuillermoz, "La Revue S.I.M. », dans Louis Laloy et al., Le tombeau de Jules Écorcheville, suivi de lettres inédites, Paris, Dorbon aîné, 1916, p. 32.

61. Laloy, La musique retrouvée, p. 138.

62. En mars 1907, Vuillermoz, regrettant le manque d'audace des dernières œuvres de Debussy, publie un article dans La Nouvelle Presse dans lequel il l'invite à écouter « les œuvres des jeunes compositeurs qu'on lui donne comme élèves " où il «retrouvera très adroitement mises en œuvre toutes les trouvailles sonores qu'il semble avoir oubliées » («Les grands concerts ", La Nouvelle Presse, 5 mars 1907, p. 1). Laloy y répond dans la Revue musicale SIM ("Les écoliers », Revue musicale SIM, 3, 4, 15 avril 1907, pp. 367-372). Voir aussi Louis Laloy, « Correspondance [de Vuillermoz] », Revue musicale SIM, 3, 6, 15 juin 1907, pp. 668-670.

63. Lettre de Jules Écorcheville à Émile Vuillermoz, 10 septembre 1914, Fonds Jules Écorcheville, citée dans Michel Duchesneau, «La Revue musicale ou le phœnix musical », Revue musicale OICRM, 4, 2, 2017, pp. 19-34 : 23, https://revuemusicaleoicrm.org/rmo-vol4-n2/revue-musicale.

64. L. L. [Louis Laloy], « Aux lecteurs », Le Mercure musical, 1, 1, 15 mai 1905, pp. 1-2.

65. Pour une étude approfondie de ces revues, voir Yoan Vérilhac, La jeune critique des petites revues symbolistes, Saint-Étienne, Publications de l'Université de Saint-Étienne, 2010 et Françoise Lucbert, Entre le voir et le dire : La critique d'art des écrivains dans la presse symboliste en France de 1882 à 1906, Rennes, Presses universitaires de Rennes, 2005.

66. Louis Laloy, "Paroles sur Claude Debussy», Le Mercure musical, 2, 5, $1^{\mathrm{er}}$ mars 1906, pp. 193-197: 195.

67. Jean Marnold, « À la Nationale », Le Mercure musical, 2, 3, $1^{\text {er }}$ février 1906, pp. 121-122.

68. Armande de Polignac, «Pensées d'ailleurs», Le Mercure musical, 1, 13, $1^{\mathrm{er}}$ novembre 1905, pp. 511-513: 511 .

69. Émile Vuillermoz, « Le dictionnaire [II]», Le Mercure musical, 2, 12, 15 juin 1906, pp. 547-555 : 547.

70. Jean Marnold, «Le scandale du prix de Rome [I et II] », Le Mercure musical, 1, 3, 15 juin 1905, pp. 129-133 et 1, 4, $1^{\text {er }}$ juillet 1905, pp. 178-180. À ce sujet, voir Gail Hilson Woldu, « Au-delà du scandale de 1905 : Propos sur le Prix de Rome au début du XX ${ }^{e}$ siècle ", Revue de musicologie, 82, 2, 1996, pp. 245-267.

71. J. M. [Jean Marnold], « Échos : Au Conservatoire », 1, 4, $1^{\mathrm{er}}$ juillet 1905, pp. 184-185: 185.

72. Louis Laloy, « Les théâtres », Le Mercure musical, 2, 21-22, 15 novembre 1906, pp. 336-340 : 339.

73. Ibid., p. 340.

74. Laloy regrettera dans ses mémoires d'avoir été aussi sévère envers Bruneau (La musique retrouvée, p. 199).

75. Pan, «Échos : Preuves », Le Mercure musical, 1, 12, $1^{\mathrm{er}}$ novembre 1905, pp. 497-499 : 497.

76. «Échos : Remerciements », Le Mercure musical, 1, 8, $1^{\mathrm{er}}$ septembre 1905, pp. 341-342.

77. Vérilhac, « La petite revue », p. 367.

78. Xavier Leroux, « Aux lecteurs», Musica, 98, novembre 1910, p. 162.

79. Charles Joly, «Chronique du mois », Musica, 35, août 1905, p. 114.

80. Gervais, « La presse illustrée », p. 458.

81. Heinich, De la visibilité, pp. 158 et 168.

82. Pour un aperçu de cette question, voir Sylvain Caron, « Les traces de l'évolution du statut des compositrices au début du XX $\mathrm{XX}^{\mathrm{e}}$ siècle dans la revue Musica », Revue musicale OICRM, vol. 4, $\mathrm{n}^{\circ}$ 2, 2017, pp. 1-18, https://revuemusicaleoicrm.org/rmo-vol4-n2/musica.

83. Duchesneau, «Composer avec l'image », p. 375. 
84. Heinich, De la visibilité, p. 49.

85. Note précédant Dominique Boulay, "Les auteurs de Miarka », Musica, 38, novembre 1905, pp. 170-171.

86. Musica, 58, juillet 1907.

87. Brétigny, « Notre tournoi : Ce que pensent MM. Massenet, Théodore Dubois et Ch. Lenepveu membres de l'Institut du Grand Tournoi International de Musica», Musica, 16, janvier 1904, pp. 243-248. Les concours de Musica semblent avoir suscité l'enthousiasme des participants. En janvier 1911, le magazine affirme avoir reçu plus de 420 manuscrits pour le concours de composition de valses lentes.

88. Cela est encore plus vrai pour l'interprétation, avec, par exemple, le numéro de juin 1908 qui publie en couverture la photo du vainqueur du Concours national de ténors non professionnels, «Louis Falandry, garçon limonadier».

89. Pasler, "A Sociology of the Apaches : "Sacred Battalion" for Pelléas ». Voir aussi Jann Pasler, "Pelléas and Power: Forces behind the Reception of Debussy's Opera », 19th-Century Music, 10, 3, 1987, pp. 243-264.

90. Edward Lockspeiser a soulevé cet aspect de la personnalité de Debussy dans sa biographie du compositeur (Claude Debussy: Sa vie et sa pensée, Paris, Fayard, 1980, pp. 259-260).

91. Duchesneau, «Composer avec l'image», p.386. Duchesneau souligne aussi que les six «Concerts d'avant-garde de Musica» lancés en 1907 ne sont en fait pas particulièrement avantgardistes et ne sont au final que peu couverts par le magazine (ibid., p. 395).

92. Xavier Leroux, «La musique au théâtre », Musica, 106, juillet 1911, pp. 126-127 : 126.

93. Mary E. Davis, Classic Chic: Music, Fashion, and Modernism, Berkeley, University of California Press, 2006, pp. 15-21.

94. Avant de prendre la barre du feuilleton musical du Temps en 1920, Vuillermoz y développe la critique cinématographique. À ce sujet, voir Pascal Manuel Heu, Le Temps du cinéma: Émile Vuillermoz père de la critique cinématographique, 1910-1930, Paris, L'Harmattan, 2003.

95. Yoan Vérilhac, «La fabrique médiatique de la postérité du symbolisme ", Problématiques et perspectives: L'Atelier médiatique de l'histoire littéraire, Médias 19, 2014, http://medias19.org/ index.php ?id=16001, consulté le 17 décembre 2020.

\section{RÉSUMÉS}

Cet article propose d'explorer les statuts accordés aux compositeurs contemporains dans les premières années du $\mathrm{XX}^{\mathrm{e}}$ siècle en France, en comparant deux périodiques au format et aux lectorats très différents : d'un côté Musica (1902-1914), premier magazine musical français lancé par Pierre Lafitte, destiné à un lectorat issu de la bourgeoisie, essentiellement féminin et pratiquant la musique en amateur ; de l'autre, Le Mercure musical (1905-1906), une " petite revue » d'avant-garde dont la ligne éditoriale et les réseaux de circulation sont à l'opposé de ceux de Musica. Il s'agit de comprendre comment le format de ces deux périodiques a influencé la façon dont est conçu et véhiculé un objet commun, soit la grandeur culturelle des compositeurs contemporains. Il ressort que Musica mise sur le vedettariat et présente presque seulement des compositeurs déjà connus, alors que Le Mercure musical met principalement en avant des artistes émergents, souvent en marge ou en conflit avec les institutions officielles, en utilisant une rhétorique avant-courrière du génie qui sera un jour reconnu. 
INDEX

Mots-clés : vedettariat, génie, presse musicale, compositeurs, France

\section{AUTEUR}

\section{MARIE-PIER LEDUC}

Doctorante en musicologie à l'Université de Montréal/OICRM et en Histoire, histoire de l'art et archéologie à I'Université libre de Bruxelles/LaM

mp1leduc@gmail.com 